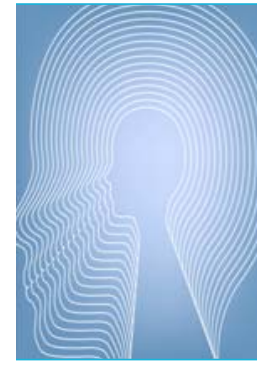

Ian B Hickie MB BS, MD, FRANZCP Executive Director

Jan Scott MB BS, MD, FRCPsych Professor of Psychological Medicine $^{2}$

Patrick D McGorry MD, PhD, FRANZCP. Executive Director, ${ }^{3}$ and Head

1 Brain and Mind Research Institute, University of Sydney, Sydney, NSW.

2 Institute of Neuroscience, Newcastle University, Newcastle upon Tyne, UK

3 Orygen Youth Health Research Centre Melbourne, VIC

4 Centre for Youth Menta Health, University of Melbourne

Melbourne, VIC

ian.hickie@ sydney.edu.au

doi: 10.5694/mjal3.1043

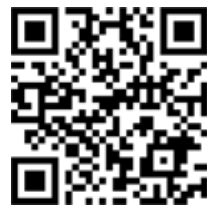

M MAA POocas:

\title{
Clinical staging for mental disorders: a new development in diagnostic practice in mental health
}

\author{
Matching the timing and intensity of interventions to the specific needs of patients
}

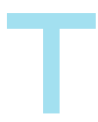

he release of the fifth edition of the Diagnostic and statistical manual of mental disorders (DSM-5) ${ }^{1}$ classification system, scheduled for May 2013, will create controversy due to the expanded range of problems now classed as mental disorders. However, in our view, it is unlikely to improve clinical care. The ultimate test for any system of diagnosis is its clinical utility. That is, does it assist clinicians to improve their selection or sequencing of treatments and enable them to make more accurate prognostic statements in keeping with newer concepts and knowledge? We propose that a refinement to traditional diagnostic practice - clinical staging - is more likely to improve clinical care and inform future research into the causes of mental disorders. ${ }^{2-5}$ Further, clinical staging draws the practice of clinical psychiatry closer to general medicine, especially with regard to chronic disease management.

In the past century, a major challenge for psychiatry was a lack of international consensus on diagnostic categories. For many decades, DSM and other international systems strived to enhance the reliability of psychiatric diagnoses. Recent field trials for DSM-5 indicate that acceptable reliability remains elusive. ${ }^{6}$ However, even diagnostic categories with modest reliability have limited validity. A major weakness is that the diagnoses refer to "categorical phenotypes" that fail to address critical differences in clinical presentations associated with age of onset or stage or course of illness. ${ }^{2-5}$ Most criteria reinforce categories derived from observations of middle-aged people with long-established stable illnesses, who have often been treated extensively by specialised mental health services. Inevitably, these map poorly onto earlier, less specific clinical presentations seen in general outpatient, primary care or community settings. They also presuppose that independent causal pathways exist for each clinical phenotype - an assumption that is not supported by contemporary family, genetic, neurobiological or risk factor research. 2,3

What are the alternatives? Twenty-first century health care places an increasing premium on personalised or stratified medicine, with the goal of delivering more tailored treatments. Consequently, there is increasing international support for applying the concepts of clinical staging, particularly for adolescents and young adults with significant mood or psychotic disorders. ${ }^{4,7}$ Given that $50 \%$ of major mental disorders begin between 15 and 25 years of age, a focus on enhanced care and novel clinical research during this critical developmental phase is a timely test of this framework. $5,8,9$

At its core, the clinical staging model recognises the full spectrum of illness experience. For example, for ischaemic heart disease, the staging model identifies individuals at risk (because of genetics, lifestyle or other risk factors), those with symptoms or related syndromes that suggest illness progression (eg, hypertension, metabolic syndrome) and those with overt evidence of cardiac disease (eg, angina). Further, treatments differ at each point on the illness continuum, with interventions with lower riskbenefit ratios being provided at earlier stages (ie, dietary change or increased exercise for the at-risk stage, and more complex medical treatments for later stages). In psychiatry, this approach also differentiates at-risk individuals (stage 0; eg, family history of severe mental disorders) from those with early subthreshold or attenuated syndromes (stage 1; eg, brief episodes of psychotic or hypomanic symptoms) from those meeting threshold diagnostic criteria (stage 2) or with established or persisting illness (stage 3). Importantly, staging suggests that disorders emerge via a limited set of overlapping and fluctuating symptom clusters (microphenotypes), some of which resolve, while others progress and stabilise into clinical presentations (macrophenotypes) that ultimately resemble more traditional diagnoses. ${ }^{2-5}$ The clinical staging model is compatible with clinical reality and can be tested for reliability, predictive validity and patterns of association with developmental epidemiology and neurobiological markers. ${ }^{7,10}$

For mental health, the critical challenge is to help all patients entering care by reducing symptoms, promoting recovery and quality of life, and preventing progression to premature death or further disability. ${ }^{8}$ The means for achieving these outcomes may be better assisted by clinical staging rather than by prematurely assigning categorical diagnoses to an evolving clinical profile. Also, rather than encouraging early use of medical interventions, the specific intent of staging models is to mimic the disease-management principles now widely applied in general medicine, which enable clinicians to target stage-appropriate interventions from early in the course of illness. Thus, for most young people entering care before the development of full- 
blown mood or psychotic disorders, the emphasis is on providing relevant information and psychological strategies (including e-mental health), reducing known risk factors (notably alcohol and/or substance misuse), minimising self-harm, promoting resilience, encouraging selfmonitoring and reviewing longitudinal course. ${ }^{8}$ In future, low-risk, evidence-based neuroprotective strategies (which are behaviourally, psychologically or medically based) may also become available.

Clinical staging revolutionised general medical practice, focusing attention on the benefits of intervening earlier in the course of life-threatening illnesses. For clinical psychiatry, it could enable appropriate matching of the timing and intensity of interventions to the specific needs of helpseeking individuals. Also, staging sheds light on the evolution of symptom networks and syndromes, ${ }^{5}$ and enhances our chances of validating specific pathophysiological pathways and of introducing more objective measures of illness risk, onset and progression. ${ }^{2}$ In medicine, the successor of clinical staging was clinicopathological staging. In the future, we hope that clinical psychiatry will also be based on the combination of objective markers of illness course and enhanced clinical care.

Competing interests: Ian Hickie was a director of headspace: National Youth Mental Health Foundation until January 2012. He is the executive director of the Brain and Mind Research Institute, which operates two early-intervention youth services under contract to headspace. He is a member of the new Australian National Mental Health Commission and was previously the CEO of beyondblue: the national depression initiative. He has led a range of community-based and pharmaceutical industry-supported depression awareness and education and training programs. He has led depression and other mental health research projects that have been supported by a variety of pharmaceutical partners. Current investigator-initiated studies are supported by Servier and Pfizer. He has received honoraria for his contributions to professional educational seminars related to depression, youth mental health and circadian-rhythms research. Jan Scott is chief investigator on a number of research studies of young people at risk of severe mood disorders and on new psychological models of treatment. She is a member of the European Network of Bipolar Research Expert Centres and the International Society for Bipolar Disorders task force on staging, and is a trustee of the International Society of Affective Disorders. She has received independent investigator awards for improving the recognition of bipolar disorders and for research on medication adherence and training clinicians in strategies for improving engagement in treatment. She has been involved on advisory boards and has been funded to attend meetings on psychosocial aspects of bipolar disorders by Janssen-Cilag, Eli Lilly, BMS-Otsuka, AstraZeneca, Lundbeck, Servier and Sanofi-Aventis. Patrick McGorry is director of headspace and also of Headstrong: National Centre for Youth Mental Health (Ireland) and he holds investigator-initiated research grant support from Janssen-Cilag, Eli Lilly, Bristol-Myers Squibb (BMS) and AstraZeneca. He also has consultancy and advisory roles with Janssen-Cilag, Eli Lilly, BMS, AstraZeneca, Roche and Pfizer.

Provenance: Commissioned; externally peer reviewed.

1 American Psychiatric Association. DSM-5: the future of psychiatric diagnosis. http://www.dsm5.org (accessed Apr 2013).

2 Hickie IB, Scott J, Hermens DF, et al. Clinical classification in mental health at the cross-roads: which direction next? BMC Med 2013. In press.

3 Scott J, Leboyer M, Hickie I, et al. Clinical staging in psychiatry: a cross-cutting model of diagnosis with heuristic and practical value. Br J Psychiatry 2013; 202: 243-245.

4 McGorry PD, Hickie IB, Yung AR, et al. Clinical staging of psychiatric disorders: a heuristic framework for choosing earlier, safer and more effective interventions. Aust N Z J Psychiatry 2006; 40: 616-622.

5 McGorry P, van Os J. Redeeming diagnosis in psychiatry: timing versus specificity. Lancet 2013; 381: 343-345.

6 Freedman R, Lewis DA, Michels R, et al. The initial field trials of DSM-5: new blooms and old thorns. Am J Psychiatry 2013; 170: 1-5.

7 Hickie IB, Scott EM, Hermens DF, et al. Applying clinical staging to young people who present for mental health care. Early Interv Psychiatry 2013: 731-743.

8 Hickie IB. Youth mental health: we know where we are and we can now say where we need to go next. Early Interv Psychiatry 2011; 5: 63-69.

9 Paus T, Keshavan M, Giedd JN. Why do many psychiatric disorders emerge during adolescence? Nat Rev Neurosci 2008; 9: 947-957.

10 Dominguez MD, Wichers M, Lieb R, et al. Evidence that onset of clinical psychosis is an outcome of progressively more persistent subclinical psychotic experiences: an 8-year cohort study. Schizophr Bull 2011; 37: 84-93. 